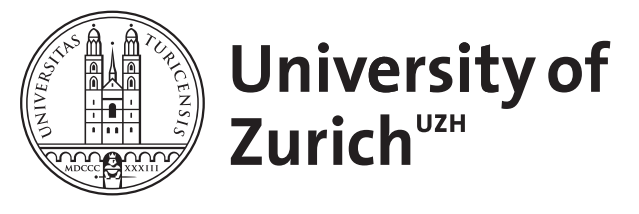

\title{
Schönes Spiel!
}

\author{
Gyr, Ueli
}

\begin{abstract}
Golfen fordert nicht nur den Spieler, sondern auch den Analytiker im Versuch, den Humor in Golferwitzen zu verstehen. Der Beitrag problematisiert zunächst den realen Spielverlauf und legt damit jene Elemente frei, auf die sich die Witze beziehen, auch mit Einblicken in internationale Aspekte. Inhaltlich überzeichnet werden hier z.B. die Hingabe an Spiel und Regelwerk, die Geschlechterdifferenz, Sexualität sowie Alter, Sterben und Tod. Unter Einbezug der heiteren Golfliteratur lassen die Witze den Golfer als eine tragische Figur erscheinen, die den Ernst des Golfspiels über den Humor abdämpft und erträglich macht. Golf erweist sich so auch als Korrektiv und Möglichkeit, das Leben und die Welt spielerisch zu begreifen.
\end{abstract}

DOI: https://doi.org/10.1515/fabula-2015-3-405

Posted at the Zurich Open Repository and Archive, University of Zurich ZORA URL: https://doi.org/10.5167/uzh-171908

Journal Article

Published Version

Originally published at:

Gyr, Ueli (2015). Schönes Spiel! Fabula, 56(3-4):289-315.

DOI: https://doi.org/10.1515/fabula-2015-3-405 
Man soll die Dinge nicht so tragisch nehmen, wie sie sind Karl Valentin

\section{Ueli Gyr}

\section{„Schönes Spiel!“}

\section{Zum Humor in Golferwitzen}

Zusammenfassung: Golfen fordert nicht nur den Spieler, sondern auch den Analytiker im Versuch, den Humor in Golferwitzen zu verstehen. Der Beitrag problematisiert zunächst den realen Spielverlauf und legt damit jene Elemente frei, auf die sich die Witze beziehen, auch mit Einblicken in internationale Aspekte. Inhaltlich überzeichnet werden hier z.B. die Hingabe an Spiel und Regelwerk, die Geschlechterdifferenz, Sexualität sowie Alter, Sterben und Tod. Unter Einbezug der heiteren Golfliteratur lassen die Witze den Golfer als eine tragische Figur erscheinen, die den Ernst des Golfspiels über den Humor abdämpft und erträglich macht. Golf erweist sich so auch als Korrektiv und Möglichkeit, das Leben und die Welt spielerisch zu begreifen.

Abstract: Golfing not only challenges the player but also the analyst who tries to understand the humour in golf jokes. This contribution problematizes the way that golf is played, revealing the elements the jokes refer to, including insights in international aspects. The jokes exaggerate, for example, gender differences and sexuality as well as age, dying and death. Golf jokes and light golf literature make the golfer appear as a tragical figure, humorously toning down the earnestness of the game. Golf thus proves to be a corrective and a possibility to understand life and the world in a playful way.

Résumé : Jouer au golf n'est pas seulement un défi pour le golfeur mais aussi pour l'analyste qui essaie de comprendre l'humour des plaisanteries sur ce jeu. Cet article problématise d'abord le cours du jeu, dégageant les éléments auxquels les plaisanteries se réfèrent, y compris des aspects internationaux. Les plaisanteries exagèrent, par exemple, les différences entre les sexes, la sexualité, l'âge et la mort. Le plaisanteries et la littérature divertissante sur le golf font paraître le golfeur comme un personnage tragique, tout en réduisant avec humour le sérieux du jeu. Le golf apparaît donc comme un correctif et offre la possibilité de comprendre la vie et le monde d'une manière ludique.

Prof. em. Dr. phil. Ueli Gyr: Universität Zürich, Institut für Sozialanthropologie und Empirische Kulturwissenschaft, email: gyr@isek.uzh.ch 
Das Golfspiel bringt vieles in Bewegung, nicht nur den kleinen Ball. Nicht alles dreht sich um ihn. Es werden auch andere Elemente aktiviert, die den Spielverlauf vom ersten bis zum letzten Schlag prägen. Sie treffen auch den Spieler, genauer: seine Befindlichkeit, der er sich irgendwo zwischen teuflischer Depression und paradiesischem Stimmungshoch aussetzt. Für Nicht-Golfer nicht nachvollziehbar, wird gleichzeitig ein zweites Spiel gespielt, mit eigenen mentalen und psychischen Herausforderungen. Dabei setzen die einen auf Technik, Talent oder Routine, während andere Glück, Leidenschaft oder gar Obsession, Sucht und Therapie heraufbeschwören. Mit einer solcherart ,total ${ }^{\text {* }}$ gerichteten Erfahrung leben Millionen von Golfern. Sie versuchen immer wieder, einen Ball auf einem eigens dafür konstruierten Golfplatz zu bewegen, das heißt, das Ziel mit möglichst wenig Schlägen über 18 oder 9 Löcher zu erreichen. Vom Abschlag durch die Fairways (Spielbahnen) über die oft als schikanös verfluchten Bunker (Sand), Wasserhindernisse und hohes Rough (ungemähte Wiese) bis hin zu den Annäherungsschlägen führen allerlei Wege und Umwege durch ein Martyrium oder über eine Himmelsleiter zum Green: Es ist pro Loch und Platz die jeweils letzte Station, bei der der Ball schließlich einzulochen (putten) ist und im besten Fall Erlösung bringt.

\section{Spielen und Erzählen}

Der allgemein bekannte Spruch „Schönes Spiel“, das man sich beim Start (oder auch unterwegs) gegenseitig wünscht, ist Ritual und Herausforderung zugleich. Es erinnert vorweg, daß das Spiel nicht nur gut, sondern außerdem auch schön sein soll, obgleich dafür keine Punkte verteilt werden. Für normabweichende ,crazy balls', das heißt komische Schläge mit gutem Resultat, gibt es inoffizielle Bezeichnungen, z.B. den ,UBS-shot‘, was soviel heißt wie ,ugly but successful'. Ein neutrales Golfspiel kann es, allgemein betrachtet, nicht geben, allein aufgrund der Tatsache, daß dieses in der Regel narrativ und wertend aufbereitet wird, ähnlich wie in diesbezüglichen Expertendiskursen, Materialgesprächen, Geschichten, Legenden, Sagen und Laiendeutungen in anderen Sportarten auch.

Zwar ist das Ganze ,nur' ein Spiel, wie ein aufmunternder Reflex auf einen schlechten Spielverlauf von Mitspielern helfend zu korrigieren versucht, doch dieses Ganze vermittelt unbestreitbar mehr und anderes. Es unterliegt zahlreichen Regeln und Etiketten, einer individuell an Turnieren erspielten Spielstärke (Handicap) sowie einem hier interessierenden Populärwissen. Golf spielen heißt nicht zuletzt, Golf auch erzählend zu bewältigen, immer wieder von neuem „Golf erzählen ist viel schöner als Golf spielen“, wie ein Bonmot pointiert. Die narrative Aufbereitung erfolgt ereignisbezogen in einer never ending story, 
zunächst für sich selber, dann für die anderen, mit denen man in einem Flight (Gruppe) gerade unterwegs ist oder war. Im Schutz der Gruppe erfährt der einzelne solidarische Zuwendung, besonders dann, wenn in Krisenmomenten nichts mehr geht. Dabei greifen die Mitspieler ein und bauen den Betroffenen mit allerlei Ratschlägen und Ermunterungen auf. Es herrscht, wiederum tragisch daher kommend, häufig verkehrte Welt. Man sagt auch bei verzogenen Schlägen z.B.: „Das hast du aber gut gemacht“, „Super, dein Ball war gerade“ oder „Such jetzt keine Länge und denk nicht zuviel“, „Wir wissen, du kannst es!“ u.a.m. Solche Aufbausequenzen dauern so lange, bis die Ordnung im Flight wieder hergestellt ist, kurz: bis man ins Spiel zurückgefunden hat und ,back on track vermelden darf, um im Jargon des Spiels zu bleiben.

Am ,Loch 19` (Scherzname für Clubhaus) vollzieht sich - immer noch in der Welt der Golfer - ein wichtiger Übergang bei einem Drink - hier wird intensiv erzählt, rekonstruiert und konstruiert, was gelaufen ist. Das Clubhaus ist zugleich der Ort, „wo wir nach der Runde unsere Wunden lecken. Das Ergebnis ist mitgeteilt, das Bag ist verstaut, die Bestellung ist aufgegeben. Und dann geschieht etwas, was wir aus dem Alltag kennen, allerdings in komprimierter

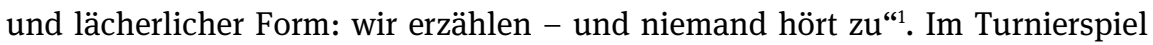
soll man das Erzählen und Besprechen tunlichst unterlassen; vor allem die Belehrungen stoßen auf und geben Strafpunkte. Selbstreferentielles Erzählen ist im übrigen nicht golfspezifisch: es läßt sich ebenso z.B. bei den Surfern, Tennisspielern, Wanderern, Bikern oder Bergsteigern beobachten.

Gemäß eigenen Beobachtungen sind es die Leader eines Flights, die unterwegs Witze erzählen, z.B. wenn ein Platz ,überbevölkert‘ ist und ein Stau entsteht. Der Herausgeber einer Witzesammlung rund um Golf meinte, es sei höchste Zeit gewesen, „daß die kursierenden Witze während Mannschaftssitzungen, Stammtischrunden, aus den Weiten des World Wide Web, auf Schulhöfen, in Clubhäusern, in Umkleidekabinen, der Playerslounge, während Mensdayrunden etc [...] gesammelt und niedergeschrieben wurde[n]“². Nach einer schlechten Runde kompensieren betroffene Spieler gerne und trumpfen mit Witzen auf, was wohl situativ der Sicherung der eigenen Position dient.

Diese Sicherung umfaßt vieles und bleibt im Kern eine individualistische Angelegenheit. Man spielt zwar in einer Gruppe, aber nicht kompetitiv gegen deren Mitspieler. Das Golfspiel stützt auf die im Handicap erlangte Spielstärke ab. Dafür sorgt ein geniales 1889 von Dr. Stableford erfundenes Zählsystem, welches jedem Spieler seinen eigenen Spielraum zuweist: Man spielt stets im

1 Bungert 2007, 14.

2 Behl 2012, Cover. 
Rahmen seiner eigenen Möglichkeiten, auf seinem Level. Das Handicap zeigt gleichzeitig an, wieviele zusätzliche Schläge dem Spieler pro Loch und Platz zustehen, die schließlich in Punkte und eine Rangierung überführt werden. So wird verständlich, daß ein Spieler mit höherem Handicap und mehr zusätzlichen Schlägen einen Spieler mit tiefem Handicap und wenig (oder keinen) zusätzlichen Schlägen durchaus schlagen kann. Unterwegs ist der Golfer mit seinem Zähler allein, sei es kämpfend, sei es verzweifelnd oder sei es beschwingt, glücksorientiert und suchend.

Vom Ball scheint eine ganz besondere Ausstrahlung auszugehen, oft mit magisch-abergläubischen Momenten durchsetzt (,komisch, mein Ball landet immer da“). Er wird nicht selten personifiziert, und gute wie schlechte Schläge werden auch ihm angelastet. Mit dem Ball muß man gemäß populärem Verständnis persönlich und einfühlsam Kontakt aufnehmen, man muß ihn kennzeichnen und mit ihm kommunizieren. Jeder Ball, so hört man auf dem Platz immer wieder, hat eine Botschaft. Sie wendet sich an den Spieler und meint aus umgekehrter Perspektive: „Schlag mich nicht, spiel mit mir!“ Schlage ich oder peitsche ich den Ball, so rächt er sich („Gott straft sofort!“). Lasse ich mich dagegen mit ihm ein und versuche ein schönes Spiel auch für ihn, belohnt er mich. Von dieser einfach gedeuteten Beziehung leben Ballkontakt und Ballresultat und zeigen eine wichtige Hierarchie: Der kleine Ball erzieht mich und nicht umgekehrt - er macht mich ganz klein und demütig. Kämpfe ich gegen ihn oder kämpfe ich überhaupt dagegen, habe ich keine Chance.

\section{Tragik und Glückssuche}

Die Belohnung für einen ,schönen`Umgang mit dem Ball ist erheblich, um nicht zu sagen zentral. Der ,fliegende Ball‘, wie der Kulturwissenschafter Ronald Lutz in einem sehr anregenden Essay gezeigt hat, führt den Spieler zur Suche nach Glück und zu Glücksgefühlen. Dabei geht es immer nur um das kleine Glück und zwar eines, das von Leichtigkeit und Flüchtigkeit gekennzeichnet ist - das Sekundenglück. Nach einem tollen oder unbeschreiblichen Schlag kann es bei den Spielern durchaus zu Luftsprüngen, Applaus oder Emotionen kommen, doch ist das Sekundenglück prinzipiell offen und nicht planbar:

„Es kommt und geht, nach uns unerfindlichen Mustern, ohne Regel, nicht feststellbar. Das aber ist die Tragik des Offenen, sie gebiert immer neue Sterne und sie lässt die Bälle immer anders fliegen als gedacht [...]. Mehr ist nicht erreichbar, alles andere ist Fiktion, die immer 
nur ein Mythos bleibt [...]. Es ist ein Spiel mit den fliegenden Bällen und es ist eine Legende vom Glück. Es ist ein Spiel um das Glück, das Spiel ist ein Glück.“3

Was viele Golfer nicht wahrhaben wollen, erweist sich analytisch als ein Grundmerkmal des Spiels: man darf dieses nicht erzwingen, es unterliegt anderen Gesetzen. Es gilt der berühmte Satz von Steven Pressfield aus dem verfilmten Golferroman Bagger Vance (USA 2000): „Du kannst dieses Spiel nicht gewinnen, du kannst es nur spielen. Ja, das ist es. Wer gewinnen will, der hat schon verloren“ (Lutz 2007, 44). Natürlich vertieft sich der Durchschnittsgolfer im Spiel nicht in analytischen Zusammenhängen der eben dargestellten Art, hat er doch ganz andere Probleme auf dem Platz, wo bekanntlich vieles ändert. Tut er dies trotzdem, so übersetzen seine Bewegungsmuster und sein Erfahrungsspeicher die Realität des Spieles auf eigene Weise. Spricht er vor dem Spiel, während des Spieles oder nach dem Spiel darüber, so enthüllt er sich ungewollt als Suchender, unterwegs nach ebendiesem Sekundenglück. Hier ist er nicht allein. Er gehört zur Gemeinschaft jener Individualisten, „die sich als Menschen neu oder anders oder überhaupt entwerfen wollen; und natürlich auch die Kopisten, die sich darin darstellen möchten“ (Lutz 2007, 45).

Wie schnell Erfolg oder Absturz unter die Haut gehen bzw. der Identität zusetzen können und wie nahe sie beieinander liegen, weiß jeder aktive Golfer. Er weiß dies spätestens, seit er die Platzreife (Prüfung für Zulassung) erreicht hat. Golfen meint immer auch ein Stück Selbstverwirklichung, hier auf besonderen Plätzen und in besonderen Konstellationen und Situationen. Beobachtet man, wie Golfer sich vor der Runde schlagmäßig auf der Driving Range (Übungsgelände) aufwärmen, stößt man zumeist auf ernste Gesichter und angespannte Körper, die etwas vom Ernst des Spiels andeuten. Die Driving Range ist ein symbolischer Tummelplatz. Er gibt auch den Kopisten eine Chance, die Suche nach dem kleinen Glück über entsprechende Inszenierungen zu proben. Fast scheint es, als müsse jeder Golfer seinen persönlichen Schlag und seine Körperbewegung zunächst auf der Matte vorführen. Die Inszenierung übersetzt den Wunsch vom Transfer auf den Platz, bleibt aber der Illusion nach wie vor verhaftet. Die Tragik des Golfers ist immer auch die Tragik des Spiels, geleitet von der Hoffnung, irgendwann doch einmal den großen Coup zu landen. Der Glaube an diesen wird hier tausendfach vorgeführt und über individuelle Inszenierungen gleichsam öffentlich simuliert - gemäß dem Motto „Nach dem Schlag ist vor dem Schlag“: nur der nächste Schlag zählt, immer wieder von vorne beginnend und mit großer

3 Lutz 2007, 42, 43, 44. 
Leidenschaft ausgetragen. Es ist eine Leidenschaft, die diesen Namen verdient, weil sie auch wirklich Leiden schafft.

\section{Golf und Humor}

Wechseln wir vom Rasen auf die Ebene der Fachliteratur über Golf, so stoßen wir bald auf die Tatsache, daß hier auffallend viele Veröffentlichungen zu finden sind: „Es dürfte nur wenige Sportarten geben, über die mehr nachgedacht und geschrieben wurde als über Golf [...]. Und 70 Millionen Begeisterte wollen in all ihren Ansprüchen befriedigt werden." ${ }^{* 4}$ Nicht alles aus der Gebrauchs- und Ratgeberliteratur rund um den Golfsport ist reine Fachliteratur, an die mit den klassischen (weltweit gelesenen) Lehrbüchern von Bob Rotella und Deepak Chopra erinnert sei ${ }^{5}$. Ein Teil des Büchersortiments hat ein zweites auffallendes Markenzeichen darin, daß dessen Orientierung recht häufig über den Humor gewählt wird. Das löst für uns allgemeine Fragen aus: Warum eigentlich Humor? Warum so viel Humor? Zufall? Wohl kaum. Die Liaison von Spiel und Humor läßt aufhorchen und drängt zu einer These, die hier zur Diskussion gestellt sei.

Man darf vermuten, daß zwischen der tragischen Figur des Golfers, der Tragik des Spiels und seiner eigenen (wie der ihm zugeschriebenen) Komik innere Zusammenhänge bestehen. Die Thesensubstanz ist einfach: Sie postuliert im Humor ein Korrektiv, demzufolge dieser den starken Anforderungsdruck des Spiels abzudämpfen versucht. Der physische, mentale und psychische Druck wird auf humoristische Art und Weise verarbeitet. Die komplexe Sache Golf soll über flotte Sprüche, Witze, Redensarten, Sprichwörter, Glossen, Anekdoten, Augenzwinkern, Schmunzeln und Ironie entschärft, das heißt auf dem Platz wie in der Literatur über eine sanftere und tendenziell heitere Vermittlungsform erträglich gemacht werden: „Golf ist, wenn man trotzdem lacht.“

Dieser Gedanke findet sich in der Golfliteratur auf Schritt und Tritt, doch wird er nicht explizit thematisiert. Ausgewählte Hinweise auf eine besondere Textsorte müssen hier genügen. So gibt sich Frank Baumanns Buch (2013) als Leidfaden für Golfer. Nachdem Matthias Brockmann (2013) auf den ganz normalen Wahnsinn des Golfens fokussiert hat, wartet er mit Geschichten auf, die der Golfplatz schrieb und meint: Wer zuletzt locht, locht am besten. Eingebettet in Amüsantes von Fairway und Green bilanzieren Kurt W. Zimmermanns Überlegungen Grundsätzliches in der Schrift Echte Golfer weinen nicht (2013). Vom

4 Kölbing/Widl 2013, 6.

5 Rotella 2004, 2007; Chopra 2003. 
Schweizer ,Golfpapst` Yves Cédric Ton-That beleben gleich mehrere Bücher den Markt, dies mit witzigen Geschichten und Anekdoten rund um die Golfregeln (Soll ich droppen oder was ... ? [2001], Soll ich Fore rufen oder was... ? [2002], Soll ich besser legen oder was... ? [2009]), wogegen das Buch Überleben auf dem Golfplatz einen nicht ganz ernst gemeinten Survival-Ratgeber anzeigt (2003), dem die heitere Sammlung Golf Zitate (2002) zur Seite steht und auch Sprichwörter sowie Aphorismen einschließt. Über Golf und die Kunst des Scheiterns denkt Eugen Pletsch nach (2009). Klaus-D. Heid/Guido Bock beschäftigen sich dagegen in dem humorvollen Büchlein Sex für Golfer (2002) damit, so der Klappentext, „wie Golf zu einem ungeahnt erotischen Ereignis wird“. Das amüsante Buch von Gerhard Bungert eignet sich explizit für jene Golfer und Golferinnen, „die einmal herzhaft über die doch wahren Geschichten am 19. Loch lachen wollen“6.

Die Breite der Palette der Schriften ist tatsächlich bemerkenswert - wer sucht, der findet im Internet über „Golf Humor“ mühelos das Passende aus 86 allein in deutscher Sprache erschienenen Veröffentlichungen mit humorvoller Ausrichtung $^{7}$. Ihre Titel erzeugen jene Spannung, die auf den hier gesetzten Strukturzusammenhang pulsiert. Sie sind griffig und direkt, wie weitere Beispiele zeigen: Echte Golfer bleiben treu, Echte Golfer fahren links, heißt es etwa, oder Cartoons für Golfer, Loch 19. Golfer zwischen Lust und Frust, Die Partherapie, Nach der Runde ist vor der Runde, Golf. Kleine Philosophie der Passionen, Golfisch. Die einzig wahre Golfsprache, Achtung Golfer. Schlägertypen in Wald und Flur, Weitund Schaumschläger. Amüsantes und Skurriles aus dem Golfalltag sowie Machen Sie ruhig so weiter ..., Golf Gaga. Der Fluch der weißen Kugel, um nur sie zu erwähnen.

Viele der eben genannten Veröffentlichungen haben inzwischen mehrere Auflagen hinter sich, sind kleinformatig, oft mit Illustrationen aufgezogen und stellen in Aussicht, daß die Leser mit amüsanten Geschichten, unterhaltsamen Lektüren, satirischen Dokumentationen, heiteren Bildern und humorvoll geladenen Regeln oder gesammelten Kolumnen rechnen dürfen. Sie helfen, das Spiel mit überspitzter Gewichtung zu positionieren. „Beim Golfen“, wie mir ein gestandener Golfer einmal erklärte, „geht es nicht um Leben und Tod - es geht um viel Wichtigeres!“ Ein weiteres und eindeutiges Indiz zuhanden der These von der Bedeutung von Humor im Golfsport liefern gezielte Internetrecherchen. Selbst über die Eingabe einfacher Kombinationen resultieren erdrückende Datenmengen.

6 Bungert 2007, Umschlag.

7 http://www.buecher.de/go/search_search/quick_search/q/cXVlcnk9Z29sZitodW1vcg==/ receiver_object/shop_search_quicksearch/ (Zugriff am 4.1.2015). 
Der Suchbefehl „Humor und Golf“ spuckt bei Google über 32 Millionen (!) Ergebnisse aus, die zu ordnen unmöglich ist und eher zur Resignation als zum Humor führen. Hier finden sich sehr unterschiedliche Formate, darunter Aufzeichnungen über Youtube, lustige Videos, Clips, Kurzgeschichten, Werbung, Comics, Cartoons, Bilder, Pins und Witze ${ }^{8}$. Inhaltsanalysen sucht man hier zwar vergeblich, doch im Mittelpunkt all dieser Humorladungen steht der Golfer als Figur, die ihre generellen Schwierigkeiten im Umgang mit dem Ball demonstrativ vorführt. Der Gewinner ist zumeist der kleine Ball, wogegen der Akteur sich oft selber parodierend in seiner Tollpatschigkeit als schwache Figur zu erkennen gibt. Sie hat viel zu kompensieren und muß daher im Wunschdenken logischerweise zu Höchstleistungen ansetzen - nicht immer lustig, und nicht immer von Erfolg gekrönt! Dieses Grundmuster der vorliegenden Kompensation typisiert narrative wie piktorale Darstellungen.

\section{Merkmale und Strukturen}

Nach der Präsentation einiger Merkmale des Golfspiels seien nun ausgewählte Golferwitze näher betrachtet und in den eben skizzierten Ereigniszusammenhang zwischen Spiel, Tragik, Glück und Humor eingebettet. Das hier genutzte Korpus von über hundert Witzen resultiert aus gedruckten Witzsammlungen im Lager der heiteren Golfliteratur ${ }^{9}$, aus Internetrecherchen ${ }^{10}$ sowie aus dem Repertoire von mündlich kursierenden Witzen. Zu bedenken ist, daß Witze immer auch Bilder, Vorurteile und typische Situationen transportieren, die von außen einstrahlen. Dazu gehört zum Beispiel die Auffassung, daß Golf gar kein richtiger Sport sei, sondern mehr eine sanfte Bewegungstherapie für ältere Männer und weiter, daß der ,Herrensport‘ teuer und langweilig sei, zudem versnobte Züge trage. Einzelne Golfclubs geben hier Gegensteuer und betreiben, wie zum Beispiel der Golfclub Duisburg, konkrete Aufklärungsarbeit. Sie versuchen konkret,

8 https://www.google.ch/?gws_rd=ssl\#safe=off\&q=humor+und+golf (Zugriff am 4.1.2015).

9 cf. z.B. Behl 2012; Friedrich 1998.

10 Die hier verwerteten Witze finden sich unter www.golfsatire.de/Golferwitze und Cartoons, wogegen die mit * bezeichneten Beispiele im Golfclub Obere Alp zwischen 2007 und 2014 von mir selber aufgezeichnet wurden. Für weitere Witzsammlungen cf. www.deecee.de/funnystuff/witze-jokes/golf-witze.html; www.sprueche-index.de/witze; www.goldisenioren.ch/aller lei/dies-und-das/golfwitze; http://witze365.de/witz-kategorie/72-Golf-Witze; www.amazon.de/ Golf-Witze-Witzesammlung-rund/dp/38442211662; www.de.findeen.com/golfwitze.html; (Zugriffe am 4.1.2015). 
den Golfsport von seinem elitären Image zu befreien und „Vorurteile, dumme Sprüche und Gelaber“ zu Gunsten von Sachinformationen zu bekämpfen ${ }^{11}$.

Doch der Humor geht auch hier eigene Wege. Zwischen Witz und Spruch, Lebensweisheiten, populärem Wissen und Wunschdenken zu unterscheiden, ist nicht einfach, und auch hat die formale Gestaltung (Länge) keinerlei besondere Merkzeichen. Sehr verbreitet und immer wieder von neuem vermittelt sind Merksprüche, die stets ein Körnchen Wahrheit enthalten. Als Beispiel diene der sehr beliebte und kürzeste Golferwitz: „Jetzt kann ich’s!“ (gemeint ist: das Spiel, das niemand beherrscht), einschließlich der Variante „Gestern“ (gemeint ist: gestern ging es noch!) oder die Frage nach dem besten Schlag, der für Alte und Junge, Männer und Frauen, Berufsspieler und Amateure, Anfänger und Gestandene, Schwerstbegabte und Untalentierte gilt. Es gibt nur einen Schlag, der alles erfüllen kann: „Der nächste“. Und: An fortgeschrittene Golfer mit Erfahrung richtet sich die Frage: „Was haben Golfplätze und Dominastudios gemeinsam? Man gibt einen Haufen Geld aus, um gedemütigt zu werden.“

\section{Hingabe an Spiel und Regelwerk}

Hingabe und bedingungslose Treue zum Spiel, gleich in welcher Intensität sie ausgedrückt werden, rekurrieren auf ein Ganzes und akzentuieren mit einer Kluft zwischen der Golferwelt und dem Alltagsleben. Dabei schimmern immer auch Vorstellungen darüber durch, wie ein gutes und ein schlechtes Spiel auszusehen hat. Der Golfer bleibt hier in der Defensive.

Golfer: „So schlecht habe ich noch nie gespielt!“ Caddy: „Was, Sie haben schon einmal gespielt?“

Bei einem Zählspiel-Turnier landet Frank im Topfbunker, aus dem er so gerade noch rausgucken kann. Erster Versuch, den Ball aus dem Bunker zu spielen; Plopp gegen die Bunkerwand. Zweiter Versuch Plopp, Dritter Plopp, Vierter Plopp, worauf sein Kumpel Markus fragt: „Du, Frankie? Hast Du die Sportart gewechselt?“ „Wieso?“ fragt Frank leicht säuerlich. „Nun“, sagt sein Kumpel, „Das sieht momentan mehr nach Squash aus!“

Während eines gemischten Turniers fragt der Amateur den Profi: „Wie gefällt Ihnen mein Spiel?“ „Es ist okay! Aber mir persönlich ist Golf lieber.“

Druck und Leistungsmessung eines ständig herausgeforderten Spielers gehen an diesem selbstverständlich nicht einfach vorbei. Als eine Art Reflex im Witz wird

11 www.golf-duisburg.de/pages/golf-ist-sch..php (Zugriff am 4.1.2015). 
auch in extremen Situationen eine klare Hierarchie markiert - das Spiel wird umgedeutet. Die Richtung ist eindeutig und meint: Golf ist Golf und Golf geht vor.

Freundin: „Golf! Immer nur Golf! Du denkst an nichts anderes. Ich packe jetzt meine Sachen und gehe zu meiner Mutter zurück!“ Golfer: „Gut, während du packst, werde ich noch ein paar Bälle schlagen.“

An einem wunderschönen Golftag sind zwei Spieler unterwegs und frönen dieser herrlichen Sportart. Als die beiden auf dem Grün des 5. Loches sind, kommt gerade auf der Strasse ein Trauerzug vorbei. Einer der beiden nimmt seine Kopfbedeckung ab und verharrt mit geneigtem Haupt. Darauf der andere Spieler: „Schöner Zug von dir, so pietätvoll innezuhalten!“ Die Antwort: „Das ist doch wohl das Mindeste, was ich meiner Frau schuldig bin!“

* Ein Ehepaar spielt gemeinsam in einem Turnier. Er spielt seinen Ball vor eine Scheune. Da sagt sie: „Ist ja nicht schlimm. Du öffnest das Tor hier, ich öffne das hintere Tor und kannst Du gut durchspielen.“ Gesagt getan. Die Frau, ein wenig neugierig, schaut durch das hintere Tor, ob ihr Mann den Ball auch gut trifft. Er trifft; nicht nur den Ball, auch seine Frau an der Schläfe. Sie bricht sofort tödlich getroffen zusammen. Der Mann hört mit dem Golfspielen auf. Viele Jahre später lernt er eine Frau kennen, die auch Golf spielt; so fängt er auch wieder an. Und wie der Zufall es will, spielen die beiden in einem Turnier gemeinsam und er plaziert seinen Ball wieder genau vor der Scheune. Da sagt die Frau: „Ist ja nicht schlimm. Du öffnest das Tor hier, ich öffne das hintere Tor und kannst Du gut durchspielen.“ „Nein“, sagt der Mann. „Das mache ich nicht noch einmal. Beim letzten Mal habe ich zwei Strafschläge bekommen.“

In übersteigerter Form erscheint die Erfüllung des Regelsystems als normativer Schlüssel, der alle beschriebenen Ereignisse außerhalb des Spiels entweder aufhebt oder aber ausblendet. Makabre Situationen können leicht entstehen. Das Spiel überlebt in unterschiedlichen Konstellationen.

Zwei Ärzte spielen auf der 15, als einer eine Herzattacke bekommt. Sagt sein Mitspieler: „Ich kann dir leider nicht helfen, denn auf dem Golfplatz greift meine Kunstfehler-Versicherung nicht. Aber ich kenne einen Kollegen, der spielt hinter uns, den werde ich holen!“ Nach einer Weile kommt er zurück, geht aufs Grün und puttet seelenruhig weiter. „Was ist mit meiner Hilfe, wann kommt sie denn?“ stöhnt sein Mitspieler. „Nun mach mal keine Hektik, der Kollege spielt gerade die 3 zu Ende. Er beeilt sich. Die anderen vor ihm lassen ihn durchspielen.“

Die Krankenschwester fragt: „Wer wird denn da operiert?“ „Ein Mann, der vor einer Stunde einen Golfball verschluckt hat“, entgegnet der Assistenzarzt. „Und wer ist das, der dort auf der Bank wartet?“ „Das ist der Besitzer des Balles. Er wartet auf den Ball, um weiterspielen zu können.“

Ein Golfer, dessen Frau verstorben ist, wird zum Gerichtsmediziner gebeten. „Ich stand am Herrenabschlag, meine Frau ca. zehn Meter vor mir am Rand des Damenabschlages. Ich schlug den Ball mit dem Driver und traf meine Frau am Kopf. Sie fiel auf der Stelle um wie 
ein Stück Holz. Ich habe keine Ahnung, wo der Ball geblieben ist.“ „Wir haben ihn bei der Obduktion gefunden“, entgegnet der Gerichtsmediziner, es war ein Top-Flite. Darüber hinaus haben wir noch einen Maxfli im Rektum ihrer Frau gefunden. „Oh“, sagt der Golfer, „das war mein provisorischer Ball.“

\section{Spannungsfelder zwischen den Geschlechtern}

Ein stark besetztes und häufig genutztes Element ist ohne Zweifel das Spannungsfeld zwischen Mann und Frau. Golfen Frauen wirklich anders, wie Matthias Brockmann meint ${ }^{12}$ ? Spielen sie zusammen, werden zum einen mögliche Animositäten, Streit und Meinungsverschiedenheiten zwischen den Geschlechtern direkt oder indirekt ausgetragen, zum anderen erscheinen sie in diskreter Form. Sie prägen je fallweise eine weibliche oder eine männliche Perspektive.

Das Ehepaar Klausi und Mausi spielt Golf. Er meckert dauernd an ihren Schlägen rum. Er schickt sie zum Pro, um besser putten zu können. Nach einer Woche ist sie zurück und schlägt bei Loch $1 \mathrm{ab}$ - „Hole in one“! Er sagt: „Hör auf mit dem Scheiss-Schlag, so lernst du nie richtig putten!“

„Das ist doch gar kein gemischter Vierer“, wundert sich ein Golfer, „das sind doch nur Männer!“ „Das schon, aber die einen können spielen und die anderen nicht!“

Eine Frau kommt vom Golfplatz glückstrahlend nach Hause. „Was ist los?“ fragt der Mann. „Liebling, stell dir vor, der Pro hat $\mathrm{zu}$ mir gesagt: ,Du hast Beine wie eine Zwanzigjährige!'“ Er brummt Unverständliches. Dann hat er zu mir gesagt: „Du hast eine Brust wie eine Dreißigjährige.“ „Und über deinen vierzigjährigen Arsch habt ihr nicht geredet?“ „Nein Liebling, kein Wort über dich!“

Peinlichkeiten werden in Golferwitzen ebenfalls strukturgebend. Junge Blondinen müssen einmal mehr als Projektionsfiguren herhalten, doch die über einen Kontroll- und Kompetenzverlust zustande gekommene Versagerleistung ist nicht exklusiv, sie trifft beide Geschlechter. Interessant ist, daß sich Peinlichkeiten hier auf Körperlichkeiten wie auf das Regelwerk beziehen. Die ,Entladung der diesbezüglichen Schwächen mutiert zum Höhepunkt.

* Eine junge Blondine hat eben mit dem Golfsport begonnen, ist begeistert und bekommt nicht genug. Ihrem Pro wird dies langsam zuviel, so daß er sie am Morgen allein auf den

12 Brockmann 2013, 82f. 
Platz schickt zum Üben. Am Abend trifft sie ihn im Clubhaus. Er fragt: „Wie war es?“ Sie meint: „Alles gut gelaufen, heute habe ich 54 gespielt, morgen gehe ich ans Loch 2.“

* Eine Blondine wird von ihrem Pro auf den Platz geschickt: „Üben, Üben, Üben - das ist das beste Rezept für dich“, wie er meint. Nach stundenlangem Üben begegnet sie dem Pro, der wissen will, wie es war. Sie sagt: „Es ging recht gut heute, aber zwischen dem ersten und dem zweiten Loch hatte ich einen Bienenstich“. Darauf der Pro: „Ich hab dir immer gesagt, daß du zu breit stehst!“

Ein Mann bittet seinen Arzt um Hilfe: „Ich fürchte, meine Frau wird taub. Immer, wenn ich sie etwas frage, bekomme ich keine Antwort.“ „Dann prüfen sie doch ihr Hörvermögen“, rät ihm der Arzt und gibt ihm Anweisungen. Am nächsten Tag spielt der Mann mit seiner Frau eine Runde Golf. Als sie auf dem ersten Grün sind und die Frau konzentriert die Linie ihres 8-Meter-Putts prüft, stellt sich der Mann fünf Meter hinter sie und fragt: „In welche Richtung bricht das Grün?“ Keine Antwort. Er geht zwei Meter näher, fragt wieder - wieder keine Antwort. Da stellt er sich direkt neben sie und sagt ganz dicht an ihrem Ohr: „In welche Richtung bricht das Grün?“ Da antwortet die Frau aufgebracht: „Zum vierten und letzten Mal: Nach links!“

Die Auslagerung golfbezogener Leiden vom Platz ins Sprechzimmer einer ärztlichen oder psychiatrischen Praxis erscheint nicht sehr häufig. Den Gang zum Arzt wählen eher Männer, doch dies bleibt ein marginales Thema, kodieren doch die kleinen oder großen Wehwehchen kaum tolle Geschichten. Als Ausnahme gibt es lediglich eine seltene, medizinisch namenlose Krankheit, „wenn einem Golfer für einen schlechten Schlag keine Ausrede einfällt“, wie Brockmann diagnostiziert ${ }^{13}$.

In einer Nervenheilanstalt sieht der diensthabende Arzt drei Irre mit Besen herumlaufen, während ein vierter lachend dabeisteht. Der Arzt fragt diesen, was da vor sich gehe, und bekommt als Antwort: „Ich habe einen kleinen Kreidekreis am Boden gezogen und demjenigen meinen Nachtisch versprochen, der als erster einputtet.“

Maier kommt zum Psychiater: „Herr Doktor, bin ich ein Mensch oder ein Tier?“ „Ein Mensch natürlich, wieso fragen Sie?“ „Jeden Tag nach der Arbeit hetze ich wie ein Hund zum Golfplatz, wenn der Ball im Bunker landet, komme ich mir vor wie ein Ochse, anschließend schufte ich bei Flutlicht auf der Driving Range wie ein Esel. Wenn ich dann spätabends nach Hause komme, fragt mich meine Frau: ,Hast du wieder Überstunden gemacht, du Rindvieh?““

„Mein Arzt hat mir geraten, mit dem Golf aufzuhören.“ „Warum? Hat er dein Herz untersucht?“ „Nein, er hat meine Scorekarte gesehen!“

13 Brockmann 2013, 91. 
Ähnlich wie die Geschichten um die jungen Golferinnen kursieren Ereignisse und Situationen bei den Senioren. Es handelt sich um eine eigene Zielgruppe, die dem Alter mit dessen klischierten Vorstellungen, Bildern und Rollen ausgesetzt ist und häufig an die Grenzen des Körpers und des Spiels gedrängt wird. Die Spannweite zwischen respektierten Golfern mit viel gelebter Erfahrung und Spätzündern, die in fortgeschrittenem Alter nach der Pensionierung neu in diesen Sport einsteigen, ist beträchtlich. Nach dem erfolgreichen Berufsleben müssen diese sich im neu gewählten Ballsport erst einfinden, d.h. zumeist hinten anstehen. Das fällt nicht immer einfach. Seniorenwitze wandeln diese Spannung häufig aus der eigenen Betroffenheit selbstkritisch um.

Drei Senioren auf der Runde. Beginnt der erste zu meckern. „Der Rasen ist aber stumpf heute!“ Der zweite mault: „Und er könnte mal wieder gemäht werden!“ Der dritte Senior: „Hört auf zu Jammern! Immerhin sind wir noch auf der richtigen Seite des Rasens!“

Georg ist 63 Jahre alt, spielt viel. Nach einer Runde sagt er zu seiner Frau: „Heute lief es wieder prima, nur meine Augen machen mir zu schaffen, Die meisten Bälle finde ich nicht.“ „Spiel doch mit meinem Bruder Peter als Caddy, der ist zwar schon 84 Jahre alt, hat aber Augen wie ein Falke.“ Am nächsten Tag stehen Georg und Peter am ersten Abschlag. Georg spielt einen 200 Meter Drive. „Hast du ihn gesehen?“ fragt Georg. „Natürlich!“ antwortet Peter. Die beiden gehen bis zu jener Stelle des Fairways, wo der Ball gelandet sein sollte, finden ihn aber nicht. Da sagt Georg: „Ich dachte, du hast gesehen, wo er gelandet ist?“ „Natürlich,“ sagt Peter, „aber ich hab es vergessen!“

Eine feuchtfröhliche Gesellschaft älterer Herren erscheint gerade im Klubhaus, als einige flotte junge Golferinnen gehen wollen. „Hallo, schönes Fräulein“, versucht einer der Kavaliere anzubandeln, „wohin geht es denn so eilig?“ „Nach Hause - unseren Omas Bescheid sagen, daß jetzt etwas Passendes im Klubhaus ist.“

\section{Sexualität und Frivolitäten}

Daß zwischen Golfsport und Sexualität allerlei Verbindungen hergestellt werden, ist allgemein bekannt. Der Satz „Hast du noch Sex oder spielst du schon Golf?“ gehört bereits zum Allgemeinwissen und führt hier zu jenen Witzen, die zwischen körperlicher Ertüchtigung und erotischem Wunschdenken zu verorten sind. Die einen meinen, daß Golf und Sex das Gleiche sei, während andere für eine Genußhierarchie einstehen oder in einer schönen Golfrunde so etwas wie einen Flirt erleben. Golf als Stimulans für Sex oder umgekehrt? Tatsache bleibt, daß es zahlreiche Witze und Sprüche über Golf gibt und recht viele von ihnen mit Sex zu tun haben. Als Erklärung wird häufig die Einsicht angeführt, „daß Golf und Sex die zwei einzigen Dinge sind, die auch dann Spaß machen, wenn man 
es nicht richtig kann“, wie Kurt W. Zimmermann anmerkt, um diese Aussage aber gleich wieder zurückzuziehen ${ }^{14}$. Demgegenüber versucht das von Klaus-D. Heid und Guido Bock herausgegebene Büchlein Sex für Golfer, den Fachjargon des Golfspiels auf die humoristische Sprache von Sexualität und Erotik umzubiegen, auch mit einer reichhaltigen Selektion von kurzen Witzen. Sie zeigen typische Situationen, Wunschdenken, Überraschungen sowie Phantasien.

* Zwei Frauen spielen Golf. Die eine macht den Abschlag - kräftig, schnell, weit - und mitten in eine Gruppe Golfer. Einer der Männer greift sich auch sofort zwischen die Beine und fällt wie ein gefällter Baum um. Die beiden Frauen eilen hinzu um zu helfen. Der arme Kerl stöhnend am Boden, die Hände immer noch zwischen den Beinen. Die eine kniet sich herunter und sagt zu dem Verletzten: „Ich bin Masseuse, vielleicht kann ich ihnen helfen und ihr Leiden lindern.“ Er lehnt stöhnend ab. Sie fühlt sich schuldig und schiebt seine Hände sanft zur Seite, öffnet vorsichtig seine Hose und fängt an, ihn im Genitalbereich zu massieren. Sein Gesichtsaudruck zeigt nach kurzer Zeit, daß es ihm schon besser geht. Auf ihre Frage, wie er sich fühle, antwortet er: „Da unten fühle ich mich großartig, aber mein Daumen tut nach wie vor höllisch weh.“

Drei Männer stehen am ersten Abschlag, da kommt eine schöne Frau und fragt: „Darf ich mich Ihnen anschließen?“ Natürlich wollen sie die Runde mit ihr spielen, doch die Frau warnt: „Ich bin Anfängerin, Sie müssen Geduld mit mir haben.“ „Kein Problem!“ sagen die drei. Die Frau schlägt vom ersten Tee ab, der Ball landet mitten auf dem Fairway. Mit dem zweiten Schlag liegt sie am Grün. Als sich der Flight dem Grün nähert, sagt die Frau: „Wenn ich den Putt einloche, wäre dies mein erstes Birdie auf einem Par 4. Wer mir dabei hilft, mit dem werde ich die kommende Nacht verbringen, und ich verspreche ihm, daß kein Wunsch unerfüllt bleibt.“ Der erste Golfer begutachtet die Puttlinie von allen Seiten und meint: „Der Ball wird sechs Zentimeter nach links brechen.“ Der zweite Golfer lotet mit seinem Putter das Grün aus und sagt: „Der Ball wird vier Zentimeter nach rechts brechen.“ Da sagt der dritte Golfer zur Frau: „Sie können den Ball aufheben - er ist geschenkt.“

Drei Damen des Golf-Clubs betreten ihre Garderobe und sehen einen nackten Mann unter der Dusche. Er bedeckt sich rasch mit einem Handtuch und flieht aus dem Raum. „Huch!“ meinte die erste, ,ich dachte schon, das wäre mein Mann.“ - „Nein, nein!“ sagt die zweite, „er ist es nicht, und es ist auch nicht mein Lover.“ - Darauf die dritte: „Es ist keiner vom Club!“

Treffen sich drei Golfer und sprechen über ihr Sexleben. Sagt der erste: „Wenn ich mein Sexleben taxieren müsste, würde ich mir Handicap 11 geben“. Sagt der zweite: „Ich bin ja nun nicht mehr der Jüngste, ich gebe mir Handicap 23“. Sagt der dritte : „Mich dürft ihr überhaupt nicht fragen, ich habe schon seit zwei Jahren keine Scorekarte mehr abgegeben!“

14 Zimmermann 2013, 9. 
Andere Witze spielen mit Einzelelementen oder setzen stärker auf Überraschungsmomente im Sinne unerwarteter Situationen und Höhepunkte, die die Auflösung bringen. Die Tatorte heimlicher oder verheimlichter Begegnungen liegen außerhalb des Clubs; andere Ordnungen gelten hier. Das Clubleben ist eine eigene Spielwiese, auf der vieles rekonstruiert wird.

Gustav besucht seine heimliche Freundin. Die Luft ist rein, ihr Mann ist nicht zu Hause. Während sie miteinander schmusen, klingelt das Telefon. Als sie wieder auflegt, fragt Gustav: „Na, wer war es denn?“ „Mein Mann. Er hat gesagt, es könnte heute etwas später werden ... er ist mit dir am Golfplatz.“

Auf seiner Golfrunde trifft Bernd eine attraktive Golferin und spielt mit ihr den Rest der Runde. Dann verabreden sie sich noch auf einen Drink im Clubhaus. Sie mögen sich immer mehr. „Willst du bei mir noch einen Kaffee trinken?“ fragt sie, und er nimmt dankend an. In ihrer Wohnung angekommen, dauert es nicht lange und sie fangen an sich zu küssen und steigen zusammen ins Bett. Irgendwann sagt er: „Ich muss jetzt nach Haus, meine Frau wird schon warten.“ Zu Hause angekommen, erzählt er seiner Frau: „Du Schatz, ich möchte dich nicht anlügen und erzähle dir, was mir passiert ist. Ich habe im Flight eine tolle Frau getroffen, die mich zu sich nach Hause eingeladen hat. Dort haben wir uns heftig geliebt, und so ist es halt später geworden. Bitte verzeih mir.“ Sie antwortet: „Liebling, du hast auch schon besser gelogen! Mach mir nichts vor, du kannst ruhig zugeben, daß du heute wieder einmal 36 Loch gespielt hast.“

\section{Zwischen Leben und Tod}

Tod im Spiel und Todeswunsch für eine andere Person finden sich in zahlreichen Varianten. Opfer entfallen auf beide Geschlechter. Die Ordnung des Spiels bleibt in jedem Fall erhalten.

Ein lang verheiratetes Ehepaar spielt eine Runde Golf. Auf dem 13. Grün hat die Frau einen 15 Meter langen Putt zum Eagle. Sie: „Wenn ich jetzt mit einem Schlag einloche, würde ich sterben.“ Er: „Liebling, ich schenke dir den Putt.“

Zwei Frauen unterhalten sich am 19. Loch ihres Golfklubs. „Hast du von Veronika gehört?“, sagt die eine. „Nein, was ist mit ihr?“ „Sie soll ihren Mann ermordet haben und befindet sich in Untersuchungshaft.“ „Wirklich? Wie hat sie es angestellt?“ „Mit einem Golfschläger.“ „Interessant! Wie viele Schläge?“

Ein Mann, der am frühen Morgen um sechs von zuhause weggegangen ist, um eine Runde Golf zu spielen, kommt lange nach Einbruch der Dunkelheit zurück. Seine Frau ist ungehalten und will nun wissen, wo er so lange gewesen sei. „Schau, ich gehe in der Früh zum Auto, und die Kiste springt nicht an. Deshalb rufe ich Walter an, damit er mich abholt. Auf dem Weg zum Golfplatz hat Walters Auto einen Defekt, und wir müssen zehn Kilometer zur nächsten Busstation gehen, um Hilfe zu holen. Als Walters Auto wieder läuft ist es kurz 
nach zehn. Als wir am Platz eintreffen, beginnt gerade das Seniorenturnier. Wir müssen zwei Stunden warten, bis wir abschlagen können.“ „Aber das war vor mehr als zehn Stunden“, murrt die Ehefrau. „Schon richtig, und wir haben anfangs auch recht flott gespielt. Doch am fünften Grün wird Walter von einem verirrten Ball getroffen. Er fällt um wie ein Stück Holz und ist auf der Stelle tot. Die nächsten dreizehn Löcher waren die Hölle: den Ball spielen, Walter schultern, den Ball spielen, Walter schultern ...“

Nach dem Ableben des Golfers muß das Golfspiel weitergehen. Diese Wunschvorstellung und die Frage, was diesbezüglich zu unternehmen bleibt, prägen auch diverse Witze aus dem Standardrepertoire. Die aktiven Golfsportler wollen wissen, wie es im Himmel diesbezüglich zu und her geht, um sich darauf einrichten zu können. Sie vernehmen, daß Gott, Jesus und Petrus sowie Pfarrer und Nonnen auch golfen, daß sie aber das, was auf Erden im Spiel getrieben wird, nicht bis ins Letzte kennen. Das Golfspiel im Himmel hat im Witz wenig Kontur, aber im Wunschdenken existiert es. $\mathrm{Zu}$ vernehmen sind allerlei Zeichen vom Himmel.

Fragt ein Golfer seinen Pfarrer: „Sie haben doch gute Beziehungen zum Himmel, können sie nicht mal für mich rauskriegen, ob man im Himmel auch Golf spielen kann?“ „Ich will es gerne versuchen“, sagt der Pfarrer, „nächste Woche nach der Kirche weiß ich mehr.“ Die Woche vergeht und die beiden treffen sich wieder. Ich habe eine gute und eine schlechte Nachricht für dich. Die gute Nachricht ist: „Es gibt ganz hervorragende Golfplätze im Himmel!“ Die schlechte Nachricht ist: „Morgen früh um 08.15h hast du Abschlagzeit!“

* Der liebe Gott schickt Petrus auf die Erde um nachzusehen zu lassen, ob da unten alles in Ordnung ist. Petrus kommt zurück und erstattet Bericht. „Chef, da unten ist soweit alles in Ordnung. Nur eine Sache verstehe ich nicht, da hauen die Menschen mit krummen Stöcken auf einen viel zu kleinen Ball und versuchen, diesen in ein Loch zu treffen.“ „Seltsames Spiel ist das“, sagt der liebe Gott, „wie heisst denn dieses Spiel?“ „Das habe ich leider nicht rausgekriegt“, antwortet Petrus, ,aber ich glaube, das Spiel heißt Scheisse!“

* Ein Pfarrer und eine Nonne spielen eine Runde Golf. Am ersten Loch verschiebt der Pfarrer einen Dreimeter-Putt und ärgert sich: „Scheiße! Daneben!“ „Aber Herr Pfarrer! Sie dürfen doch als Gottesmann nicht so fluchen“, weist ihn die Nonne zurecht. Am nächsten Loch liegt der Pfarrer nur zwei Meter vom Loch entfernt - und schiebt den Ball vorbei. „Scheiße! Daneben!“ brüllt er wutentbrannt. Die Nonne wird richtig böse: „Herr Pfarrer, wenn Sie noch einmal fluchen, soll sie der Blitz treffen. “ Am nächsten Loch liegt der Pfarrer nur einen Meter neben der Fahne und wieder schiebt er den Putt vorbei und schreit zornig: „Scheiße! Daneben!“ In diesem Moment öffnet sich der Himmel, ein Blitz zuckt heraus - und trifft die Nonne. Da hört der Pfarrer eine Stimme aus dem Himmel: „Scheiße! Daneben!“ 


\section{Sagenhafte Geschichten - auch lustig?}

Wer sich die Frage aufgibt abzuklären, ob auch moderne Sagen zur golfbezogenen Humor- und Witzkultur im eben dargestellten Sinn etwas beitragen, wird kaum schnell fündig. Zwar gibt es Beispiele, die auf die Entwicklung einzelner Sagenelemente deuten, doch läßt ihre geringe Zahl keine Verallgemeinerungen zu. Ihnen fehlen heitere Momente - an deren Stelle machen sich vielmehr Merkwürdigkeiten und Skurriles am Rand von Witzen bemerkbar, die nicht alle $\mathrm{zu}$ herzlichem Lachen führen. Es ist wohl davon auszugehen, „daß Witze und moderne Sagen eine Nähe zueinander aufweisen und je nachdem, wie sie erzählt werden, entweder der einen oder anderen Kategorie zuzuschlagen sind“"15. Pointenähnliche Merkmale scheinen auf, wie das nachfolgende Beispiel erweist:

Beim Golfspiel, als es mal nicht so klappte, hat mir ein Flightpartner erzählt, er habe neulich Golf gespielt, und dabei beobachtet, wie im Flight vor ihm ein Spieler permanent schlechte Abschläge gemacht habe. Offenbar verzweifelt, habe dieser bei Erreichen des nächsten Wasserhindernisses (z.B. ein See in der Mitte des Golfplatzes) wutentbrannt seine Golftasche samt Schlägern in das Wasser geworfen und sei davon gestapft. Dann habe er umgedreht, sei ins Wasser gestiegen, habe die Tasche wieder herausgezogen, aus einer Seitentasche den Autoschlüssel gezogen und die Tasche wieder im Wasser versenkt. Dann sei er davongefahren. Die Tasche war aus teurem Leder und habe ein Set teurer Schläger enthalten. ${ }^{16}$

Bei der Geschichte von der Golftasche im Wasserhindernis handelt es sich um eine Zusendung über E-Mail, die auch deshalb wertvoll ist, weil ihr Urheber am 26. Mai 2009 dazu einen Kurzkommentar abgab. Er führte aus, daß er die Geschichte zuletzt in Brunnthal bei München von einem Freund im Golfclub gehört habe, dem das gleiche einige Wochen zuvor passiert sei. Komischerweise, setzte er selber dazu, habe er diese Geschichte schon mindestens dreimal gehört - „einmal von einem amerikanischen Geschäftspartner, der über eine Golfrunde in Miami erzählte“ (ebd.).

Unter dem Titel Golf kann ätzen hat Rolf Wilhelm Brednich eine motivverwandte Version dieser Geschichte bereits 1997 in einem neuseeländischen Club registriert und 2004 in einer interessanten Sammlung als Nr. 91 veröffentlicht ${ }^{17}$. Nach einer schlechten Runde mit Ballverlust landet der letzte Ball eines Spielers abseits in einem Teich. Darauf eskaliert das Geschehen, er dreht durch.

15 Frdl. Mitteilung von Rolf Wilhelm Brednich (Mail vom 24.11.2014).

$16 \mathrm{http} / /$ www.sagen.at/texte/gegenwart/deutschland/Golftasche.html.

17 Brednich 2004, Nr. 89-91 (freundlicher Hinweis von Hans-Jörg Uther, Göttingen). 
Jetzt kann der Unglückliche seinen Zorn nicht länger zurückhalten: Ihm entfährt ein lauter Fluch, voller Wut schultert er seine Golftasche, läuft zu dem Teich und schmeisst sie im hohen Bogen dem Ball hinterher. Danach entfernt er sich, ohne sich noch einmal umzusehen, grusslos in Richtung Parkplatz. Seine Mitspieler schauen sich an und beenden die Runde ohne ihn. Als sie gerade die letzten Putts spielen, sehen sie ihren Freund zurückkommen und in den Teich waten, um seine Golftasche zu finden: Darin war nämlich sein Autoschlüssel!

Typologisch läßt sich dieser Erzählstoff bei den Rachemotiven einordnen: Es ist im ersten Beispiel die Rache an Material und Gerät und erinnert an Wutentladungen von Tennisspielern, die bei schlechtem Spielverlauf oder Fehleinschätzungen des Schiedsrichters das Racket zerstören. Im zweiten Beispiel (Nr. 91) verstößt der Akteur gegen die hoch gewertete Etikette des Golfspiels und wird für die unzureichende Selbstkontrolle bestraft. Atypisch wirkt der definitive und radikale Abbruch des Golfspiels, wo dieses doch in Wirklichkeit eigentlich immer wieder von neuem beginnt.

Was ein Golfball vermag, Nummer 89 in der Sammlung von Brednich, erzählt die Geschichte von einem Soldaten, der im afrikanischen Benin mit seinem Golfball einen Vogel getroffen habe, der seinerseits an die Frontscheibe eines startenden Düsenjägers geprallt sei und so einen Absturz verursacht habe. Dabei seien weitere drei Flugzeuge zerstört und so die fliegenden Streitkräfte Benins ausgelöscht worden, dies mit einer Schadensumme von 30 Millionen Dollar. Einmal mehr steht hier die Allmacht des kleinen Balles an - sie wirkt hypertrophiert. Spekulativ stellen sich auch unterschwellige Verbindungen zu überragenden Spielstärken ein, die in der Golfersprache mit Namen von Vögeln besetzt sind: Birdie, Eagle und Albatros.

Preiswertes Sportgerät (Nr. 90) behandelt eine Sequenz von finanzstarken Japanern, die in Neuseeland gerne Golf spielen. Eben haben sich vier Japaner neu ausgerüstet und gehen auf die Runde. Nach der Rückkehr wollen sie ihre Golftasche zurückgeben, doch der Pro-Shop ist bereits geschlossen. So lassen sie ihre Golftaschen einfach vor dem Clubhaus stehen in der Meinung, die Geräte seien nur geliehen, was ihnen sehr billig vorkam. In Wirklichkeit hatten sie sie gekauft. Die reichen Japaner haben ein Verlustgeschäft abgewickelt, ohne es zu merken. Sie sind keine wahren Golfer.

Ein eigenes Motiv für sagenhafte Geschichten gibt zu guter Letzt das ,Hole in One‘ ab, das heißt jenes Spiel, das vom Abschlag mit einem einzigen Schlag aufs Green und direkt ins Loch geht. Das kommt in Form eines außergewöhnlichen Schlags ab und zu vor, während eine häufig dazu erzählte Geschichte diesen Spielzug gerne mit dem Tod verknüpft. Katja Doubeks Lexikon merkwürdiger Todesarten (2000) führt ein solches Beispiel aus Massachusetts vor - es ist die unglaubliche Geschichte eines 79jährigen Golfers, dem dies widerfahren ist: „Der 
begeisterte Hobbygolfer Emil Kojek hatte am 11. November 1994 das Loch mit einem Schlag getroffen und war unmittelbar danach von Stolz und Freude überwältigt zusammengebrochen. Die eilig herbeigerufenen Aerzte konnten nur noch seinen Tod feststellen“, wie die Autorin vermerkt ${ }^{18}$. Im Denken vieler Golfer gibt es nichts Schöneres als einen Tod auf dem Golfplatz. Kaum zufällig trägt ein Buch mit den von Experten bestimmten 50 schönsten Golfplätzen der Welt den Titel Play Golf Before You Die (Santella 2006). Sie erscheinen als kleine Paradiese.

\section{Weltsport und angelsächsisches Potential}

Durch die Fokussierung auf germano-geprägte Beispiele aus der humorgeladenen Literatur mag vielleicht der Eindruck entstanden sein, daß diese sich zu einer Witzkultur zusammenfügen, die einmalig sei und ihresgleichen suche. Dieser Eindruck täuscht und bleibt zu korrigieren, haben wir es doch mit einer Sportart zu tun, die sich inzwischen in vielen anderen Räumen und Spieltraditionen etabliert hat - Golfsport repräsentiert längst auch Weltsport. In dessen Rahmen drücken sich Eigenheiten, Übereinstimmungen und Bedeutungswerte von international verbreiteten Golferwitzen gleichfalls aus. Sie beziehen ihre Mischungen irgendwo zwischen mondialer Verschweißung und kulturgeprägten Erzähltraditionen, bis hin zu Clubrepertoires. In Ermangelung wissenschaftlicher Analysen seien vorläufig einige Merkpunkte festgehalten.

Stimmt die Annahme mondialer Basiselemente für das Golfspiel, für seine Praxis sowie für seinen Bedeutungswert, bliebe dies auch in Witzen und der Art von Humor in einzelnen ,Golferwelten` zu erfragen, nicht zuletzt, um mögliche Wechselbeziehungen $\mathrm{zu}$ erkunden. Produktion, Rezeption und Diffusion von Golferwitzen zu kontextualisieren und $\mathrm{zu}$ historisieren führt weiter: Von der Betrachtung der Golfpraxis anderer (nicht deutschsprachiger) Länder sind mögliche Zusammenhänge über die Bedeutung und eine allfällige Verbreitung der Textsorte Witz zu erwarten. Daß ihre Popularität auch statistisch über die Zahl der Einwohner pro Golfplatz und als relationale Dichte zu sehen ist, leuchtet ein. Die Positionierung im europäischen Vergleich ist aufschlußreich. Die Statistik zeigt für das Jahr 2011, daß Island mit 4.545 Einwohnern pro Golfplatz an erster Stelle liegt, gefolgt von Schottland (9.797), Irland (10.979), Wales (19.745), Schweden (21.789), England (28.282), Österreich (55.629), der Schweiz (84.211),

18 Doubek 2004, $145 f$. 
Deutschland (114.246) und Italien (218.705), um nur einige Länder zu erwähnen ${ }^{19}$. Im Ranking der Verteilung von Golfplätzen in Europa nehmen die Länder des Vereinigten Königreichs unübersehbar Spitzenpositionen ein. Es drängt sich daher auf, die mit dem Golfsport am stärksten verbundene angelsächsische Witzkultur analytisch ins Spiel zu bringen.

Zu einem solchen Vorhaben legitimiert auch der Befund, daß Golferwitze im germanischen wie im angelsächsischen Bereich mit gleichen oder ähnlichen Motiven kursieren, oft fast deckungsgleich sind. Daraus leitet sich die Vermutung ab, daß zahlreiche germano-zentrierte Golferwitze angelsächsischen Ursprungs sind - ,Entlehnungen', auf welchen Wegen solche auch immer zustande gekommen sein mögen. Internetrecherchen bestärken indirekt einen Zusammenhang mit Verweisen auf ein bemerkenswertes Erzählpotential. Die Suchmaschine www.google.de verzeichnet zum Beispiel über die Eingaben von „Golf Jokes Humor“ 1.280.000 Ergebnisse, von „Humor and Golf“ 32.300.000, von „Golf Jokes UK“ 17.700.000 sowie von „British Golf Humor“ 1.250.000 Ergebnisse - im Vergleich mit den Suchergebnissen aus dem deutschsprachigen Raum mit strukturell wesentlich mehr Einträgen ${ }^{20}$.

Am kleinerem Maßstab überprüft, erbringt weiter auch die Suchmaschine Amazon aussagekräftige Resultate, hier unter dem Aspekt von Sammlungen herausgefiltert. www.amazon.co.uk weist für „Humor and Golf“ 863 in englischer Sprache verfaßte Bücher aus ${ }^{21}$, das heißt, wie oben vermerkt, gegenüber deutschen Buchveröffentlichungen mit einer größeren Produktion auf dem Markt stehend. Eine Bestätigung ähnlicher Relationen zeigt sodann die Suchmaschine www.worldcat.com mit nicht weniger als 1442 Einheiten für „Humor and Golf“. Davon entfallen 1269 auf englischsprachige sowie 37 auf deutschsprachige Buchtitel, um nur sie zu nennen. Ein Blick auf die bei WorldCat herausgefilterten Buchbestände zeigt eines sehr deutlich ${ }^{22}$, daß nämlich Sammlungen von Golferwitzen auf angelsächsischer Seite offenbar länger zum Traditionsbestand gehören und sehr viel stärker vertreten sind als das, was sich in deutschsprachigen Gebieten im Vergleich fassen läßt. Die Registrierung der am gleichen Ort erfaßten Veröffentlichungen verteilt sich auf die letzten Jahrzehnte mit eindrücklichen

$19 \mathrm{http} / /$ de.statista.com/statistik/daten/studie/6914/umfrage/einwohner-pro-golfplatz-ineuropa-nach-laendern/ (Zugriff am 4.1.2015).

20 vgl. www.google.de mit allen hier erwähnten Resultaten (Zugriff am 4.1.2015).

21 http://www.amazon.co.uk/s/ref=nb_sb_noss?url=search-alias\%3Dstripbooks\&fieldkeywords=humor+and+golf (Zugriff am 4.1.2015).

22 https://www.worldcat.org/search?q=kw\%3Ahumor+and+golf\&qt=results_page (Zugriff am 4.1.2015). 
Raten von Titeln, nämlich 284 (2010-15), 454 (2000-09), 352 (1990-99), 128 (1980-89), 46 (1970-79) und 47 (1960-69).

\section{Internationaler Aufschwung und Diversifizierung}

Vor 1960 von einer Textsorte humoristischer Buchveröffentlichungen zu sprechen verbietet sich. Jahrzehntelang dominierten hier Einzelmeldungen, wogegen von einem Aufschwung ab den 1970er Jahren auszugehen ist, dann verstärkt mit einem auffallenden Produktionsschub ab etwa 1990. Kritisch sei angemerkt, daß wir es hier mit Indikatoren zu tun haben, die nur grobe Verteiler vorgeben. Sie besagen nichts über die Wege, welche Golferwitze zwischen angelsächsischer und germanischer Spieltradition realiter gegangen sind. $\mathrm{Zu}$ den Kennzeichen angelsächsischer Golferwitze gehören mit Sicherheit ihre thematische Vielfalt und die Diversifizierung von Formaten. Bücher mit Witzsammlungen scheinen sehr beliebt zu sein - es gibt hier auch ,Bestseller‘, die sich seit langen Jahren halten konnten und vereinzelt (Phillips 2001) über fünf Millionen mal verkauft wurden ${ }^{23}$.

Auf der Ebene der Kategorien, über welche angelsächsischer Golferhumor durchschlägt, sind neben den ,klassischen“ Witzen auch allerlei Kurzgeschichten, Cartoons, Sprüche, Weisheiten, Zitate und Bilder, YouTube, Clips, Videos und Pins zu erwähnen. Sie zu überschauen, ist unmöglich - von den Redundanzen ihrer Inhalte ganz zu schweigen. Es ist kein Zufall, dies eine Vermutung, daß die Erzähl- und die Witzforschung das vorliegende Terrain bisher noch nicht betreten hat. Ein Blick auf die z.B. vom Soziologen Christie Davies (2011) betriebene Humorforschung sowie die in der Zeitschrift International Journal of Humor Research seit 1988 gesichteten Jahrgänge bestätigt bald, daß die golferische Witzkultur monographisch bisher nicht abgehandelt wurde und weiterhin empfindliche Forschungslücken vorliegen.

Wie nahe deutschsprachige und englischsprachige Versionen ausgewählter Witze liegen können, zeigt ein exemplarischer Vergleich. Die konstituierenden Elemente erscheinen in leicht abgeänderter Form, aber die Grundstruktur und die ausladende Pointenvorbereitung bleiben erhalten.

Fred was playing off the sixth tee at the Royal Quebec Club. The fairway of the sixth needed some skill because it ran alongside the road. But Fred sliced the ball badly and it disappeared over the hedge bordering the road. So he put another ball down and took the penalty. He was having a beer after the game when the pro joined him in the bar. "Excuse

23 Weitere Buchtitel finden sich im Literaturverzeichnis. 
me Fred, but was it you who sliced this ball into the road at the sixth this morning?" "Yes, but I took the penalty." "That's as may be. But you might be interested to know that your ball hit und killed a small boy on a tricycle; the tricycle fell in the path of a Mountie on a motorcycle. He skidded and was thrown through the window of a car, killing the nun at the wheel. The car then swerved into a cement mixer which wasn't too damaged but had to veer slightly and in doing so ran into the local school bus with such an impact that it sent it flying through the window of the St Lawrence shopping centre. At last count from the hospital there are thirteen people dead and seventy-nine people seriously injured." The golfer turned a deathly shade of white and said, "What can I do?" "Well, you could try moving your left hand a little bit further down the shaft", the pro advised.

Nach einer besonders schwachen Runde Golf unterließ es ein bekanntes Clubmitglied, ins Clubhaus zu gehen und wollte gleich nach Hause. Als er über den Parkplatz zu seinem Auto ging, stoppte ihn ein Polizist und fragte ihn: „Haben Sie ungefähr vor zwanzig Minuten vom sechzehnten Tee abgeschlagen?“ „Ja“, antwortete der Golfer. „Haben Sie zufällig Ihren Ball gehookt, so daß er über die Bäume außerhalb des Golfplatzes ging?“ „Ja, das habe ich. Woher wissen Sie das?“ fragte er. „Nun ja“, sagte der Polizist sehr ernst, „Ihr Ball flog hinaus auf eine Schnellstrasse und durchschlug die Windschutzscheibe eines Autos. Das Fahrzeug geriet außer Kontrolle, kollidierte mit fünf anderen Fahrzeugen und einem Feuerwehrauto. Die Feuerwehr konnte nicht zum Einsatzort kommen und das Gebäude brannte nieder. So, was beabsichtigen Sie in dieser Sache zu tun?“ Der Golfer dachte sorgfältig nach und erwiderte: „Ich denke, ich werde meinen Stand ein wenig schließen, fester greifen und den rechten Daumen weiter runter nehmen.“

\section{Repertoires und Kategorienbildung}

Auf Seiten angelsächsischer Repertoires werden Golferwitze nicht unbesehen irgendwo abgelegt, sondern über einfache Kategorien gebündelt bzw. mit Hilfe von weiteren Stichwörtern und ähnlichen Motiven definitiv plaziert. www.golfjokes.co.uk illustriert dieses Darstellungsprinzip beispielhaft ${ }^{24}$. Die Kategorien heißen z.B.: Caddies, Cartoons, Dictionary, Excuses, Famous Golfers, Golf Quotes, Golfing Laws, Golfing Lists, Golfing Truths, Her Indoors, Life \& Death, Miscellaneous, OneLiners, Religion \& Golf, Rule Exceptions, The Golf Pro, Woman Golfers, X Rated Golf, You scored?!!? Bereits die auf unterschiedlichen Kriterien abstützenden Kategorien lassen erkennen, daß wir es hier (und auch bei der ,Auflösung durch weitere Witze) mit motivlichen, akteurbezogenen sowie sequentiellen Ausgliederungen zu tun haben.

Diese schlagen auch über andere Kategorienbildungen durch. www. golfjokes.com typologisiert unter „Great Golf Jokes“ zum Beispiel Funny Jokes/

24 www.golfjokes.co.uk (Zugriff am 4.1.2015). 
One Liners/Cliches and Truth/Lists/Dirty Jokes/Poems/und Top 10 Jokes. Am gleichen Ort führt ein Verteilersystem zu weiteren Sites, konkret zu Funny Golf Videos, Golf Quotes, Golf Gags, Golf Cartoons und anderes mehr. Einzeiler, Sprüche und Zitate von Prominenten erfreuen sich offenbar besonderer Beliebtheit, wie darauf ausgerichtete Sammlungen (Clean Golf Jokes, Funny Stories and One-Liners sowie Golf One-Liners and Other Short Funnies) aufzeigen.

Einzeiler und Zitate fallen kurz aus, sind oft personalisiert und bringen allerlei Golferfahrungen und Weisheiten auf den Punkt, wie einige Beispiele verdeutlichen. Sie sind aufzufinden in den websites von www.golfjokes/com/ quotes und www.guy-sports-com/humor/sports_golf.htm.

- "Golf is a game where the ball always lies poorly and the player always lies well" (anonymous)

- "The more you play it the less you know about it" (Patty Berg)

- "It takes hundreds of good golf shots to gain confidence but only one bad one to lose it" (Jack Nicklaus)

- "My golf is improving. Yesterday I hit the ball in one!” (Jane Swan)

- "If you think it's hard to meet new people, try picking up the wrong golf ball" (Jack Lemmon)

- "The only time my prayers are never answered is on the golf course” (Billy Graham)

Ob es innerhalb der oben erwähnten Kategorienbildung Präferenzen oder Hierarchien gibt, wäre im Detail zu überprüfen. Zusammenfassend läßt sich ausführen, daß die angelsächsische gegenüber der deutschen Witzkultur mit Blick auf Golf wesentlich differenziertere Ordnungssysteme entwickelt hat. Der Distributionsvorgang angelsächsischer Witze dürfte über die Weltsprache Englisch vermehrt auch informell verlaufen.

\section{Fazit und Schlußbemerkungen}

1) Golf, um nun $\mathrm{zu}$ einigen generellen Bemerkungen überzugehen, ist ein zugleich einfaches, kompliziertes sowie geheimnisvolles Spiel. Man kann es selbstverständlich auch ohne vertiefende Reflexion und Deformation spielen, doch wer den sprichwörtlich gewordenen, allumfassend wirkenden Golfvirus einmal empfangen hat, wird ihn nicht so schnell wieder los. Er befeuert gleichsam wie ein Motor eine mitlaufende Disposition, die darauf ausgerichtet 
ist, das eigene Tun im Spiel immer wieder von Neuem zu ergründen und individuell zu vermessen. Gerade weil dies kaum je richtig gelingt, entläßt dieser Stimulus den infizierten Golfer nicht mehr - er will nicht nur bis zum Lebensende spielen, sondern auch im Himmel auf schönen Plätzen weitermachen. Golf, dies sei damit ausgedrückt, ist eine ernste Angelegenheit, über die nachzudenken sich lohnt.

„Golf ist eine wunderbare, spielerische Möglichkeit, die Welt, das Leben und das Sein zu begreifen. Die Hindus nennen unsere Wirklichkeit Leela, was übersetzt ,das kosmische Spiel' bedeutet. Auch für Quantenphysiker und andere Mystiker [...] ist alles, was wir als Realität betrachten, ohne wirkliche Substanz. Golf ist somit ein Spiel im Spiel des Lebens.“25

Dieses ,Spiel im Spiel des Lebens' erreicht exakt das, was auch der Durchschnittsgolfer an Erfahrungen, Erfolg und Leiden realiter durchzustehen hat und als ,Golf-Weisheiten' beziehungsweise als ein Stück Lebensschulung verinnerlicht, unabhängig, ob er sie weiter thematisiert oder nicht.

Golferwitze setzen eine von der Forschung bisher nicht genutzte eigene Kategorie von heiteren Elementen zusammen. Sie sind relativ spät in die einschlägigen, nunmehr auch über das Internet abrufbaren Verzeichnisse von Sportwitzen eingedrungen. Man darf vermuten, daß ihr Auftritt mit der realen, ebenfalls späten Entwicklung und Popularisierung zusammenhängt, die ab etwa Mitte in den 1990er Jahre einsetzten. Dabei ist zu beachten, daß sich der einstige ,Nobelsport' stark verändert hat und boommäßig expandiert. Der Frauenanteil ist mit $40 \%$ aller Aktiven gewichtig, und auch bei Kindern und Jugendlichen erfreut sich Golfen einer großen Beliebtheit. Ein Blick auf mögliche formale Strukturen der Witze erweist, daß diese von kurzen dialogischen Abläufen geprägt sind, wobei das Spannungsfeld zwischen Mann und Frau für die Konstruktion von humorvollen Geschichten und Pointen allgemein am ergiebigsten zu sein scheint. Die Ausmachung von Positionen und Hierarchien hat inzwischen diverse Erzählmuster und Inhalte entwickelt.

2) Die Schauplätze und Tatorte, die in den hier untersuchten Witzen genannt werden, sind aufschlußreich und relevant. Sie verweisen auf einen fast exklusiven, kleinen Bewegungsradius, spielen doch die meisten humoristischen Geschichten mitsamt ihren Pointen auf dem Golfplatz, im Clubhaus, im Umkleideraum, oder aber sie sind ausgelagert im Himmel, in der Kirche, am Grab sowie vereinzelt in der Arztpraxis oder im Spital zu finden. Kaum überraschend entzünden sich die meisten Geschichten auf den bekanntermaßen ereignisreichen und ,sensiblen' Stellen und Etappen auf dem Platz, darunter zum

25 Pletsch 2009, 43. 
Beispiel Abschlag, Sandbunker, Wasser, Rough und insbesondere dem Green. Damit sind wir bei den Golfern angekommen. Als Handlungsträger oder besser als Figuren werden sie typisiert. Sie agieren in ihren Rollen, Handlungsmustern und Phantasien. $\mathrm{Zu}$ ihnen kann angemerkt werden, daß sie sich streng im Rahmen der vorgegebenen Regeln bewegen. Zwar treten Alter, Generationenkluft und Technik zugunsten von Spielfreude, Regeltreue und auch Leistung etwas zurück, aber Sexualität, Frivolitäten und kompetitives Spiel bestimmen nach wie vor beliebte Handlungsrahmen.

Analog zu ihrer inneren Struktur wird Leistung im Spiel in einem sehr viel stärkeren Ausmaß als individuelle Leistung taxiert, oft auf ironische oder parodistische Weise übersetzend. Sie lenkt so von ständig mitgezogenen Ängsten, im Spiel vor den anderen zu versagen, ab. Im Hintergrund, witzig angelehnt, steht diese Angst, auch wenn der als Druck erlebte und so häufig erwähnte Spielfaktor mehr verdrängt als ausgetragen wird. So wird auch verständlich, warum echte Golfer niemals weinen, wie der Titel einer originellen Schrift von Kurt Zimmermann (2013) diese Erfahrung umschreibt. Auch ein verpatztes Spiel bleibt ein Spiel. Im Fall des Golfspiels belastet dieses den Spieler, um ihn gleichzeitig zu entlasten - er gerät unter Druck und wird befreit, denn:

„Golf ist die einzige gesellschaftlich akzeptierte Spielform des permanenten Misserfolges. Darum ist es auch so populär geworden. In einer Zeit, in der bereits die Unterschreitung des Vorjahresergebnisses um 0,25 Prozent für den Rausschmiss aus dem Unternehmen genügt, ist Golf für uns Leistungsträger ein letztes Refugium der Unschuld geworden. Hier kann man richtig entspannt versagen." ${ }^{26}$

3) Im ernsten Spiel entspannt versagen? Ist das die Formel? Was beim ersten Blick als Widerspruch daher kommt, erweist sich bei genauerem Hinschauen als beruhigendes Korrektiv und als versöhnliche Entlastung. Sie gelten nicht dem Flight auf dem Platz mit 18 Löchern, sondern mir allein. Hier bin ich für alles verantwortlich, nicht wie z.B. im Tennis, wo der Partner auf der anderen Seite des Netzes steht und auch fleißig mitschaufelt - für sich oder gegen mich, für mich oder gegen sich, je nach Spielverlauf. Dieser übergibt mir in jedem Fall das Spiel. Dabei eingeschlossen ist aber auch seine Funktionalität, unabhängig davon, ob ich im einzelnen realisiere, daß Spiel und Spieler tragische Substanzen enthalten. Wenn ich diese spielerisch einsetze und mit Humor, einer von der Art, wie er sich in den Golferwitzen manifestiert, aufmische, empfange ich allerlei Weisungen, schön weiter zu tun, andernfalls bestraft mich der kleine Ball sofort. Von solchen Glücksmomenten und ,Strafaktionen“ handeln die Golferwitze ganz

26 Zimmermann 2013, 16. 
allgemein, auch wenn ihr Humor nur Oberflächen erreicht. Dennoch läßt sich die Unterscheidung von guten und schlechten Golferwitzen daraus ableiten. Gute Golferwitze, um das Positive aller Tragik zum Trotz herauszustellen, streuen nach oben und nach unten: ihre wahren Botschaften kommen im Himmel wie auf dem Rasen an. Man darf sie weiterreichen.

\section{Literatur}

Baumann, Frank: Single in 365 Tagen. Ein Leidfaden für Golfer. Gockhausen 2013.

Behl, Benedict (Hg.): Golf Witze. Witzesammlung rund um Golf. Kindle 2012.

Brednich, Rolf Wilhelm: Pinguine in Rückenlagen. Brandneue sagenhafte Geschichten von heute. München 2004.

Ders./Boenisch, Max: Golfen in Neuseeland. Ein Führer zu den schönsten Country Courses. Berlin 2009.

Brockmann, Matthias: Wer zuletzt locht, locht am besten. Geschichten, die der Golfplatz schrieb. Stuttgart 2013.

Bungert, Gerhard: Loch 19. Golfer zwischen Lust und Frust. Stuttgart 2007.

Chopra, Deepak: Golf for Enlightenment. The Seven Lessons for the Game of Life. London 2003.

Davies, Christie: Jokes and Targets. Bloomington 2011.

Doubek, Katja: Lexikon merkwürdiger Todesarten: Seltsame Spielarten und Formen des Exitus von A wie Amoklauf bis Z wie Zyankali. Frankfurt am Main 2000.

Drew, Simon: Golf: Fairway Fables. Woodbridge [2013].

Exley, Helen (Hg.): A Round of Golf Jokes. New York 2007.

Exner, Justin J.: 501 Excuses for a Bad Golf Shot. Naperville, Ill. 2004.

Friedrich, Gerhard: Die besten Golfer-Witze. Wien/München 1998.

Heid, Klaus-D./Bock, Guido: Sex für Golfer! München 2002.

Kölbing, Alexander/Widl, Gerhard: Golf! Machen Sie ruhig so weiter ... München 2007.

Lutz, Ronald: Der fliegende Ball. Eine Legende vom Glück. In: kuckuck. notizen zur alltagskultur. glück 22, 1 (2007) 41-45.

Penicks, Harvey: Golf-Weisheiten. Das kleine rote Buch. München 2009.

Phillips, Bob (ed.): A Classic Collection of Golf Jokes \& Quotes. Eugene 2001.

Phillips, Dilwyn: Golfing Jokes. Talybont, Ceredigion 2010.

Pletsch, Eugen: Endlich einstellig! Golf und die Kunst des Scheiterns. Stuttgart 2009.

Pressfield, Steven: The Legend of Bagger Vance: Golf and the Game of Life. New York 1995.

Röhrich, Lutz: Der Witz. Figuren, Formen, Funktionen. Stuttgart 1977.

Rotella, Bob: Golf is a Game of Confidence. London 2004.

Ders.: The Golfer's Mind. Play to Play Great. London 2007.

Santella, Chris: Play Golf Before You Die. Von Experten empfohlen. Die 50 schönsten Golfplätze aus aller Welt. Stuttgart 2006.

Stewart, Sheila and Ron: 500 All Time Funniest Golf Jokes, Stories \& Fairway Wisdom. Phoenix 1996.

Ton-That, Yves-Cédric: Soll ich droppen oder was ... ? Amüsante Geschichten zu den Golfregeln 1. Zürich 2001.

Ders.: Soll ich Fore rufen oder was ... ? Amüsante Geschichten zu den Golfregeln 2. Zürich 2002. 
Ders.: Golf Zitate. Die schönsten Zitate, Sprichwörter und Aphorismen rund um den Golfsport. Zürich 2002.

Ders.: Überleben auf dem Golfplatz. Zürich 2003.

Ders.: Soll ich besser legen oder was ... ? Amüsante Geschichten zu den Golfregeln 3. Zürich 2009.

Zimmermann, Kurt W.: Echte Golfer weinen nicht. Amüsantes von Fairway und Green. München ${ }^{7} 2013$.

Zullo, Allan: Golf is a Funny Game. Kansas City 2008. 